\title{
CONf-971066--1
}

\section{COMPENSATION FOR MISSING SENSORS IN A REAL-TIME MEASUREMENT BEAMFORMER SYSTEM}

\author{
Timothy F. Gee \\ Robert Collier \\ Kirk Everman \\ Eva B. Freer \\ G. R. Wetherington, Jr. \\ Oak Ridge National Laboratory \\ P.O. Box 2008 \\ Oak Ridge, TN 37831-6007 \\ RECEIVED \\ DUL 281997 \\ OSTI \\ Gerald Tarasek \\ Carderock Division/Naval Surface Warfare Center \\ Code 1925, Bldg. 15 \\ Bethesda, Maryland 20084-5000 \\ Kevin Pepe \\ Planning Systems Incorporated \\ 7923 Jones Branch Road \\ McLean, Virginia 22102

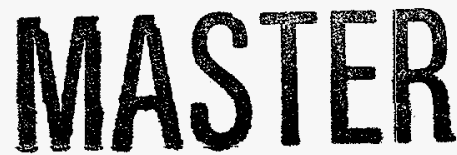

\section{DISCLAIMER}

\begin{abstract}
This report was prepared as an account of work sponsored by an agency of the United States Government. Neither the United States Gnvernment nor any agency thereof, nor any of their employees, makes any warranty, express or implied, or assumes any legal liability or responsibility for the accuracy, completeness, or usefulness of any information, apparatus, product, or process disclosed, or represents that its use would not infringe privately owned rights. Reference herein to any specific commercial product, process, or service by trade name, trademark, manufacturer, or otherwise does not necessarily constitute or imply its endorsement, recommendation, or favoring by the United States Government or any agency thereof. The views and opinions of authors expressed herein do not necessarily state or reflect those of the United States Government or any agency thereof.
\end{abstract}

Work performed for Carderock Division/Naval Surface Warfare Center, Bethesda, Maryland, under Interagency Agreement Number 1350-1350-A1 and prepared by Oak Ridge National Laboratory, Oak Ridge Tennessee 37831-6285, managed by Lockheed Martin Energy Research Corporation for the U.S. Department under Contract DE-AC05-960R22464. 


\section{DISCLAMIER}

Portions of this document may be illegible in electronic image produets. Images are produced from the best available original document. 


\title{
COMPENSATION FOR MISSING SENSORS IN A REAL-TIME MEASUREMENT BEAMFORMER SYSTEM
}

\author{
Timothy Gee ${ }^{1}$, Kevin Pepe ${ }^{2}$, Robert Collier ${ }^{1}$, Kirk Everman ${ }^{1}$, \\ Eva Freer ${ }^{1}$, Randall Wetherington ${ }^{1}$, and Gerald Tarasek ${ }^{3}$ \\ ${ }^{1}$ Oak Ridge National Laboratory, Oak Ridge, Tennessee \\ ${ }^{2}$ Planning Systems Incorporated, McLean, VA \\ ${ }^{3}$ Carderock Division/Naval Surface Warfare Center, Bethesda, Maryland
}

\begin{abstract}
In the system described in this paper, an array of hydrophones with a fixed geometry is used to make accurate underwater acoustic measurements over a large frequency range. The beamforming coefficients used to weight each sensor have been optimized over the full frequency range to provide the desired accuracy for a constant beam width and high noise reduction for the ocean environment. These coefficients must be developed for numerous angular beam widths and for several steering angles, requiring considerable time (several hours) to generate off-line using parallel digital signal processors. To make the number of coefficients manageable, the array symmetry has been utilized. When sensors are missing due to inevitable failures, this symmetry no longer exists, and a new method is required to handle the arbitrary array geometry. Also, failures can occur unexpectedly, making it unreasonable to redesign the coefficients for changes in array geometry. This paper describes a compensation method that has proven successful in regaining the desired amplitude level of the beam response without modifying the existing sensor coefficients.
\end{abstract}

\section{INTRODUCTION}

The acoustic measurement facility USNS HAYES (T-AG 195), instrumented with high gain arrays (HGAs) and real-time beamforming and signal processing systems, is the U.S. Navy's unique signature-measurement facility for performing radiatednoise trials on all quiet submarine classes. Precision sound pressure level (SPL) measurements are required to determine shipbuilder noise specification compliance, ship-signature degradations, and acoustic quieting effectiveness. Although there are a large number of sensors in an HGA, sensor failures can compromise SPL accuracy unless proper correction is applied.

Each HGA is a cylindrical array of hydrophones comprised of a ring of vertical line arrays (staves). Frequency-domain beamforming [1] is performed on each HGA to produce multiple fixed-width beams that are steered at the moving test vessel. The beamforming is performed in two stages. The first stage produces beams using the vertical staves separately. The second stage uses

Oak Ridge National Laboratory is managed by Lockheed Martin Energy Research Corp. for the U.S. Department of Energy under contract DE-AC0596OR22464

This work was funded by Carderock Division/Naval Surface Warfare Center. the resulting stave beams as a ring of sensors to produce a beam with a three-dimensional spatial response. This separability greatly reduces the computational complexity and the number of unique beamformer weights. However, the use of a small number of weights makes the occurrence of missing sensors difficult to correct. The same beamforming weights used for a stave with all good sensors must also be used for a stave with missing sensors. Also, the symmetry of the array is exploited in that weights are stored for only a portion of the $360^{\circ}$ azimuthal steering range. Therefore, it is not feasible to incorporate missing sensor compensation into the beamforming weights.

A method of measurement correction has been developed that compensates for missing sensors without changing the beamforming weights. The correction method consists of two operations. The first, stave-beam renormalization, is used for staves that have a limited number of missing sensors. Renormalization of individual stave beams produced by the first beamforming stage is performed to improve the normalization and shape of the beam created by the second stage. The second operation, stave doubling, is beneficial when a stave has too many missing sensors to be aided by stavebeam renormalization and must be removed from the beam computation. The beamforming weights of opposing staves are complex conjugates so that their imaginary responses to an input plane wave cancel one another. Doubling the input of the stave opposite a missing stave improves the array's power measurement while introducing some phase error. The phase error does not affect SPL measurement but can affect time series data recovered from the beamformed data.

Our compensation method has been successfully integrated into the HGA measurement system and has been shown to be effective at correcting the system's measurements when several sensors are missing. The analysis and implementation of these two compensation operations are discussed below with comparisons to other considered techniques.

\section{BACKGROUND}

\section{A. System Description}

An HGA measurement system has been developed to acquire accurate acoustic signature measurements and images from U.S. Navy submarines. The HGA system enables precise measurements of noise radiated from quiet submarines whose acoustic signature is significantly lower than the ocean ambient levels. The system is used in acoustic trials to measure and identify noise deficiencies that may adversely affect the vessel's stealth mission. Data collected 
from acoustic trials are used to characterize the ship's signature over all operating conditions, determine the ship's detectability, and determine ship-builder specification compliance. In addition, data measured through the HGAs are utilized to initiate studies for future advanced quieting efforts to ensure that the acoustic emissions from U.S. submarines remain at the lowest levels in the world. The system was developed between 1990 and 1995 as part of the Naval Surface Warfare Center / Naval Sea Systems Command, Acoustic Measurement Facilities Improvement Program (AMFIP). The HGA measurement system is installed and currently operational aboard the USNS HAYES (T-AG 195).

The HGA measurement system consists of multiple sub-arrays to produce high gain measurements over a large frequency range. This paper is concerned with apertures 1 and 2 , which cover the two lower frequency bands. These arrays are geometrically similar, where the sensor spacing for aperture 2 is half that for aperture 1 . The geometry of one array is illustrated in Fig. 1. These two arrays are termed the space frame arrays because of their open construction. Each array consists of 16 staves, where each stave is a vertical line array consisting of 20 hydrophones. The staves are arranged in a ring, making the whole array a cylinder. The hydrophone stave design employs a unique construction technique in which the stave cable passes axially through the center of the hydrophone ceramic to provide an omnidirectional radial response and very accurate sensor positioning.

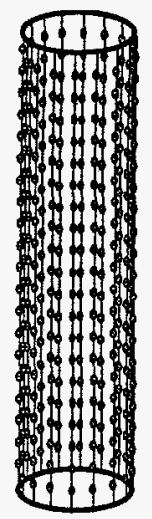

Fig. 1. Schematic representation of one of the two space frame arrays.

The hydrophone data are processed continuously in real time using an Fast Fourier Transform (FFT) beamformer to efficiently produce multiple beams over the desired frequency range. The measurement beam coefficients are generated before run-time and are optimized to provide high array gain in the ocean environment and a near constant beam width across the entire frequency coverage. Array gain is the ratio of the array signal-to-noise ratio and the omni-directional sensor signal-to-noise ratio [2]. "Beam width" refers to the size of the angular region that has an approximately flat magnitude response and is usable for making acoustic measurements. This part of the beam is called the beam's "measurement region". The system dynamically steers the measurement region at the test vessel or at a portion of the test vessel to quantify the radiated noise while attenuating other surrounding noise.

\section{B. Problem}

The harsh ocean environment and repeated handling of the hydrophone staves during deployment and retrieval operations can cause individual sensor failures in the space frame arrays. A sensor fails when its signal response becomes unacceptable, or it is too noisy to be used. In these cases, the sensor is removed from the beam calculation and considered a "missing" sensor.

Sensor losses can perturb the directional response of the array from its desired response and also yield measurement beam amplitude errors. For typical cases, the loss in array gain is minimal; however, the loss in measurement accuracy can be significant. To ensure that the required measurement accuracy is maintained, the missing sensors must either be physically replaced with operational sensors or compensated in the beamformer. Sensor failures can occur during an acoustic trial, in which case sensor replacement is usually not a cost-effective option.

\section{Solution}

To enable the investigation, an existing simulation tool was modified to analyze proposed compensation techniques. The tool can calculate the magnitudes, phases, and array gains of beam patterns. It allows the user to remove any sensors from the simulation and compare compensated and uncompensated beam responses. All of these actions can be controlled from a graphical user interface.

From the analysis, two compensation techniques were chosen. These were then implemented for real-time operation. The first is an additional filtering stage for intermediate beam data. This corrects amplitude errors caused by missing sensors. The second is the doubling of certain stave arrays to correct for whole staves that are missing. These compensation techniques restore the measurement beam amplitude accuracy and will be described below.

\section{TWO STAGE BEAMFORMER}

The HGA beamformer uses two stages to separate the elevational and azimuthal beamforming. The two stages for a space frame aperture are illustrated in Fig. 2. Each stage is a frequencydomain dot product with an individual dot product of complex values at each frequency bin. The inputs to each dot product are the narrowband sensor values and the narrowband coefficient values. In Fig. 2 the $f$ index indicates the frequency bin, $v$ is the vertical index (1 to 20), and $h$ is the horizontal index (1 to 16).

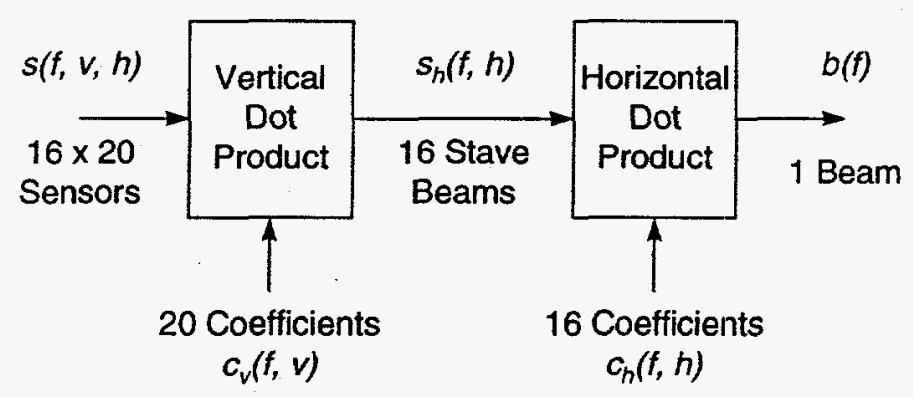

Fig. 2. Frequency-domain beamforming is performed using two dot-product stages. 
The inputs to the first stage are all of the sensor values and the vertical coefficients. In the first stage, there is a separate dot product for each bin of each stave array. Thus, 16 stave-beam values are created per bin. These stave beams and the horizontal coefficients are the inputs to the second stage. In this stage, there is one dot product per bin. The result of the second stage is called a "spot beam" because its vertical and horizontal discrimination make its measurement region an oval spot.

The two stages in Fig. 2 are also represented by $(1) . C_{v}(f)$ is a 20-element column vector of complex vertical-beamforming coefficients at frequency $f$, and $C_{h}(f)$ is a 16-element column vector that contains complex horizontal-beamforming coefficients. $S(f)$ is a 20 by 16 matrix in which each column contains the sensor data for a stave. The result of the two stages is $b(f)$, which contains the beam data for a given frequency. Equation (1) has extra parentheses to indicate the order of the real-time operations.

$$
b(f)=\left(C_{v}(f)^{T} S(f)\right) C_{h}(f)
$$

As can be seen, $b(f)$ depends on sensor availability, coefficients, and frequency. The coefficients for a given frequency vary according to the beam steering and the beam width. For this reason it is impossible to simply use the location of the missing sensors to perform a recalibration for all combinations of steering, frequency, and beam width.

Also, the system's exploitation of array symmetry makes the loss difficult to compensate. As can be seen in (1), the vector of vertical coefficients is used for every stave array. This approach assumes that all staves have the same geometry. It is impossible, within the constraints of the existing processing implementation, to have vertical coefficients that specialize to each stave.

There is a similar reuse of the horizontal coefficients $C_{h}(f)$. These coefficients are only pre-calculated for the azimuthal angles $0^{\circ}$ to $12^{\circ}$ in $2^{\circ}$ increments. Coefficients for steered angles between these intervals are obtained during run-time by interpolation. The pre-calculated range is only slightly greater than half of the angular distance between staves as is indicated by Fig. 3. This figure is a top-view of one of the space frame arrays, in which each stave appears as one sensor.

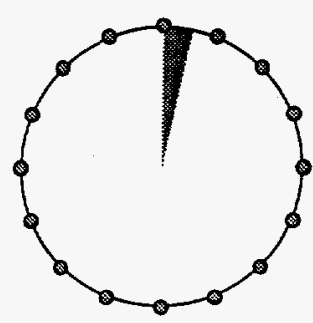

Fig. 3. Range of pre-calculated azimuthal coefficients $\left(0^{\circ}\right.$ to $12^{\circ}$ ) is indicated by shaded region.

The system has an azimuthal steering range of $360^{\circ}$ which is achieved by using the symmetry of the cylinder. The range of $-12^{\circ}$ to $0^{\circ}$ is obtained by reversing the order that the coefficients are applied to the ring. The rest of the azimuthal steering range is obtained by shifting the location of the coefficients around the ring. Each rotational shift by a stave is a $22.5^{\circ}$ shift in steering angle. As was the case for the vertical coefficients, it is not possible to design the coefficients to handle the missing sensor geometry because of their reuse.

Reuse of vertical and horizontal coefficients greatly reduces requirements for run-time storage and memory bandwidth. However, even if the run-time issues were ignored, eliminating coefficient reuse would increase coefficient generation time and storage by over an order of magnitude. Then coefficient generation would require days instead of hours. Also, specializing the coefficients to all of the missing sensor locations would require calculating new coefficients for every change in array geometry, and such changes might occur anytime during an acoustic trial.

Because of the time needed to regenerate beamforming coefficients and the run-time considerations mentioned above, the considered methods did not involve modifying the beamforming coefficients. Also, since the system was completely developed and tested, the desire was to modify the existing structure as little as possible.

\section{DISMISSED COMPENSATION METHODS}

\section{A. Sensor substitution}

One considered method involved replacing missing sensor data with that from an adjacent sensor. In simulations of this approach, the beamforming routine had an input for each sensor, where some sensor data was used more than once. The replacing sensor data had approximately the correct magnitude, but the phase was in error. The size of this error depended on the angle of the incoming plane wave in relation to the missing sensor and the sensor that supplied the replacement data. This angle is shown as $\alpha$ in Fig. 4. As $\alpha$ increases, the time (phase) offset increases, and the error caused by the substitution increases.

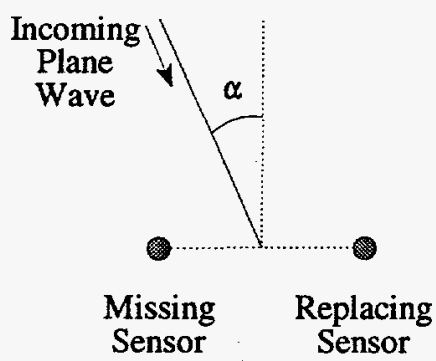

Fig. 4. Angle of incidence made by incoming plane wave and line perpendicular to the segment between the missing sensor and the replacement sensor.

In the case of a missing sensor, the substitution was made from a sensor on the same stave. The replacing sensor was either directly above or below the missing sensor. In this case, $\alpha$ was a vertical angle. Since the vessel is close to the stave's horizon, $\alpha$ remains small for the source signal, which improves the measurement response. However, the noise arrives at all angles, so the overall noise reduction is weakened. In simulations using this approach, the amplitude accuracy was good within the measurement region of the beam, but the noise floor rose considerably.

In the case of a missing stave, the data from a whole adjacent stave was used to replace the missing stave. Because the target vessel can be at any azimuthal angle, $\alpha$ can have any value for the 
source signal. This caused amplitude reduction in parts of the measurement portion of the beam pattern because of the phase error.

Sensor substitution has additional problems if several adjoining sensors are missing. For these reasons, sensor substitution was not considered to be a good solution.

\section{B. Partial delay-and-sum beamforming}

Another method simulated was the simplification of the beamforming coefficients for part of the array. Delay-and-sum beamforming [1] was used for problem staves, while the regular coefficients were used for good staves. The delay-and-sum coefficients were equal magnitude or shaded by a simple windowing function, and the phase was simply $\vec{k} \cdot \vec{r}$ where $\vec{k}$ has magnitude $2 \pi / \lambda$ and has the opposite direction from which the beam is pointing. $\vec{r}$ is a vector pointing to the sensor from the center of the stave array. The coefficient magnitudes did not vary with frequency so that compensation could be simplified. As expected, the vertical beam width varied with frequency in the delay-and-sum staves. Although only some of the staves used delay-and-sum coefficients, the coefficients in the horizontal stage were not equally weighted, and a few delay-and-sum staves could have a large impact on the resulting spot beam. This caused the overall beam response to lose its desired vertical beam width.

\section{RENORMALIZATION}

\section{A. Description}

Renormalization is done by evaluating the beam response with missing sensors and using the offset at the beam's center to shift the response back to $0 \mathrm{~dB}$. This offset is indicated in Fig. 5. This shift is a function of frequency, beam width, steering angle, and the location of the missing sensors

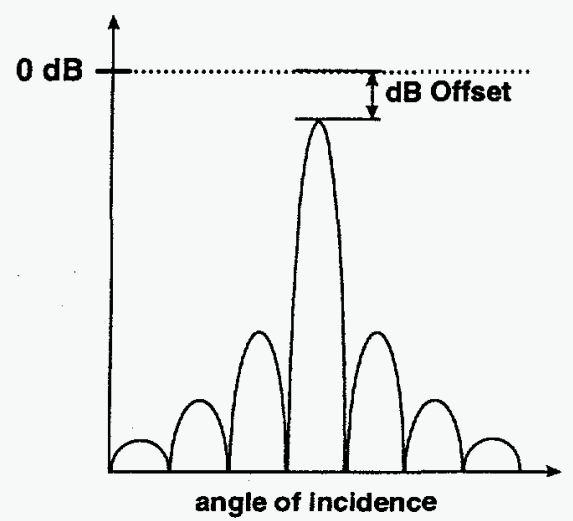

Fig. 5. Renormalization involves evaluation of the beam pattern's center and shifting it to $0 \mathrm{~dB}$ at every frequency bin.

Two renormalization approaches were considered. The first is spot-beam renormalization, where the spot beam is the output of the second dot-product stage. This process is indicated by (2), in which $R_{s p o t}(f)$ is a scalar compensation value that is dependent on frequency, elevational and azimuthal steering, and elevational and azimuthal beam width.

$$
b(f)=\left(\left(C_{v}(f)^{T} S(f)\right) C_{h}(f)\right) R_{s p o r}(f)
$$

The second approach is stave-beam renormalization. This consists of renormalizing the 16 stave beams, which are the outputs of the first dot-product stage. This process is represented by (3) where $R_{\text {stave }}(f)$ is a 16 by 16 diagonal matrix in which the non-zero entries are the scaling factors for the stave beams. $R_{\text {stave }}(f)$ is dependent on frequency, elevational steering, and elevational beam width.

$$
b(f)=\left(\left(C_{v}(f)^{T} S(f)\right) R_{\text {stave }}(f)\right) C_{h}(f)
$$

There are benefits to both approaches, but it would have been impractical to implement both given processing and storage limitations. Each approach restores the amplitude level of the spotbeam center provided no staves are missing. Stave-beam renormalization cannot compensate a stave that has all of its sensors missing. Spot-beam renormalization does aid the case of a missing stave. The primary benefit of stave-beam renormalization over spotbeam renormalization is that it improves the shape of the spot beam. This is because stave-beam renormalization compensates the intermediate values. It scales the stave beams to appropriate levels before they are input to the second dot-product stage. Since the scaling associated with spot-beam renormalization is applied after beamforming, it does not affect the shape of the final beam response.

Stave-beam renormalization had benefits from an implementation standpoint. Since the system had dynamic memory available, but little bandwidth for additional processing, it made sense to implement the normalization using a pre-calculated lookup table. The set of precalculated values necessary for stave-beam normalization is much smaller than that of spot-beam renormalization because of its independence of the azimuthal steering.

Given the benefits of improved beam response and smaller lookup table, stave-beam renormalization was selected. The problem created by a missing stave will be addressed later in the paper.

\section{B. Implementation}

The compensation values are computed off-line and stored in files that can be loaded into a dynamic memory lookup table during run-time. The compensation values are calculated at all bin frequencies and every $0.1^{\circ}$ over the full vertical steering range. For a given stave, the compensation values act as a frequency filter since they vary with frequency. The frequency-domain beamforming has a fixed amount of overlapping time samples between FFT records which are needed to implement overlap-save convolutions [3]. To avoid circular convolutions, the compensation filters are fixed to a certain impulse response length. In our case, truncating the impulse response length of the compensation filters caused very little error. The filters are stored in the lookup table as frequency-domain values so they can be applied directly between the dot-product stages.

For the cases simulated, the difference between complex and magnitude stave-beam compensation results was insignificant. The imaginary part of the stave scaling factor was found to be small compared to the real part, so using the magnitude was a very good approximation. Therefore only the magnitude of the frequencydomain values are stored.

The run-time implementation requires a considerable amount of memory, but needs little additional on-line processing. The lookup table needed in the implementation requires nearly 100 megabytes; 
however, it could be reduced by using a coarser sampling of steering angles. Even with its large size, the table can be created in under 5 minutes, making it possible to change compensation factors quickly if sensors fail during a trial.

\section{Performance}

An example of the improvement in measurement accuracy is shown in Fig. 6 . In these plots, there were 25 missing sensors. The vertical sensor spacing is $0.68 \lambda$, and the horizontal (chord) spacing is $0.63 \lambda$. The locations of the missing sensors are identified by the black squares in Fig. 7. The elevational steering is on the horizon, which is perpendicular to the stave arrays, and the azimuthal steering is at $0^{\circ}$ which is in the direction of stave 16 . Fig. 6 shows the uncompensated and compensated plots of a horizontal cross-section of the three-dimensional beam response. This beam is designed to be $77^{\circ}$ wide in the azimuthal direction, where the width is defined by the angles where the response goes below $-2 \mathrm{~dB}$. The vertical beam width is $9^{\circ}$ although it cannot be seen by the plots. In the uncompensated beam, the response varies between about -4 and $-2 \mathrm{~dB}$, whereas the response of the compensated beam is close to $0 \mathrm{~dB}$ except at the edges of the measurement region. This is a desirable horizontal response for making measurements.

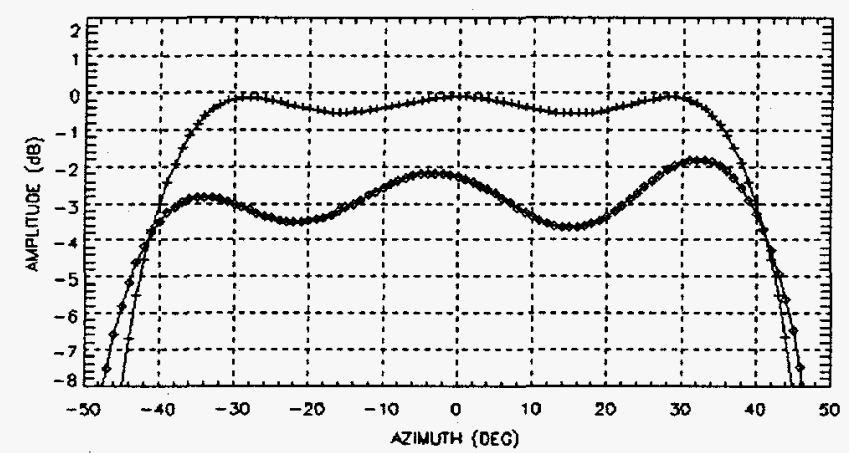

Fig. 6. Azimuthal beam responses for 25 missing sensors. ( $\diamond)$ uncompensated, $(+)$ stave-beam renormalization.

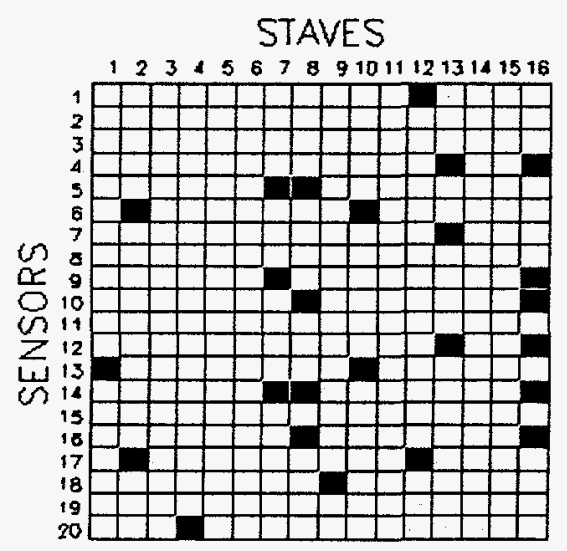

Fig. 7. Grid of missing sensors for Fig. 6. Each square is the status of a sensor. White is present, and black is missing. Each column corresponds to a stave.

\section{STAVE DOUBLING}

\section{A. Description}

Stave-beam renormalization works well as long as there are not a large number of sensors missing on any one stave array. If many sensors are missing on one stave, the stave-beam response can be very different from the desired shape. In that case, boosting the response by normalizing the center to $0 \mathrm{~dB}$ increases the problem because it increases the effect on the entire array's response. Also, the horizontal coefficients may weight this stave's measurement heavily, causing the problem stave to have a large impact on the total array response.

In the above case, it is better not to use the problem stave. In our simulations we have found that the measurement accuracy can be increased by removing the problem stave and doubling the weight of the stave on the opposite side of the cylinder. The reason this is beneficial is related to the form of the horizontal coefficients. The horizontal coefficients for the space frame arrays are designed such that coefficients that are on opposite sides of the ring are complex conjugates.

The responses from a plane wave for two opposing staves are indicated in Fig. 8. $s(f)$ is the frequency-domain signal observed at the center of the array, and $s_{1}(f)$ and $s_{2}(f)$ are the signals received at staves 1 and 2. At a given frequency, the responses of opposing staves have equal and opposite phase offsets from the signal seen at the center of the ring. Since the horizontal coefficients for these staves are complex conjugates, the weighted sum of these two staves has the same phase as the center of the array. This is illustrated in (4), where $b(f)$ is the weighted response of the two opposing staves, and $c_{1}(f)$ and $c_{2}(f)$ are the coefficients for those staves. Since the weighted sum of each pair of opposing staves is in phase with the array center, the response of the entire ring is in phase with the array center.

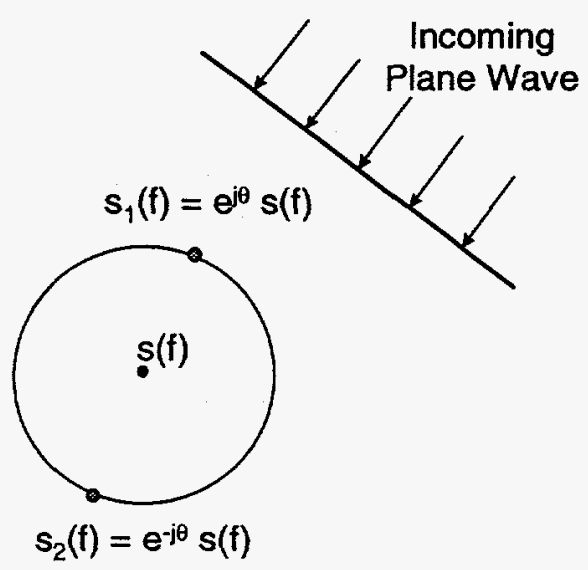

Fig. 8. The responses of opposing staves to an incoming plane wave have equal and opposite phase offsets from the response observed at the array center.

$$
\begin{aligned}
b(f) & =s_{1}(f) c_{1}(f)+s_{2}(f) c_{2}(f) \\
& =e^{j \theta} s(f) c_{1}(f)+e^{-j \theta} s(f) c_{1}^{*}(f) \\
& =2 s(f) \operatorname{Re}\left[e^{j \theta} c_{1}(f)\right]
\end{aligned}
$$


This section is concerned with the horizontal dot-product stage, but it is worth noting that the vertical coefficients also have complex-conjugate symmetry about their phase reference, which is the center of each stave array. Thus, when all sensors are present, the resulting spot beam has the center of the cylindrical array for its phase reference. Since apertures 1 and 2 are concentric, they have the same phase reference. This design enables successive beam FFT records from apertures 1 and 2 to be reconstructed into a time series.

If we continue with our two stave model, we can suppose that one of the pair is missing. This will occur if the whole stave is missing or if it has so many sensors missing that we do not want to renormalize it. The resulting response after doubling the remaining stave is shown in (5).

$$
\begin{aligned}
\hat{b}(f) & =2 s_{1}(f) c_{1}(f) \\
& =2 e^{j \theta} s(f) c_{1}(f) \\
& =2 s(f)\left\{\operatorname{Re}\left[e^{j \theta} c_{1}(f)\right]+j \operatorname{Im}\left[e^{j \theta} c_{1}(f)\right]\right\}
\end{aligned}
$$

Here we see that the response is the same as before except that the imaginary part that canceled in (4) is doubled in (5). This causes $\hat{b}(f)$ to have a different phase from $b(f)$ and to have a larger magnitude. The error in the response to an ideal noiseless plane wave is $j 2 s(f) \operatorname{Im}\left[e^{j \theta} c_{1}(f)\right]$. Note that for delay-and-sum beamforming with or without shading, $\operatorname{Im}\left[e^{j \theta} c_{1}(f)\right]=0$, so the error should be small when the phase of the coefficients are similar to delay-andsum coefficients. Also note that this one stave is added to the sum of the other staves which will have the phase of the array center. In beam simulations, stave doubling has proven beneficial at improving the amplitude with only a small effect on phase.

\section{B. Performance}

Fig. 9 shows an example of the improvement made by stave doubling combined with stave-beam renormalization. As in the previous example, the vertical spacing is $0.68 \lambda$ and the horizontal spacing is $0.63 \lambda$. Also, the horizontal and vertical beam widths are once again $77^{\circ}$ and $9^{\circ}$. The steering is on the horizon and at the same azimuthal angle as stave 16 . This time there are 34 missing sensors whose locations are shown in Fig. 10. The uncompensated response has a large amount of ripple and varies between -5 and -2 $\mathrm{dB}$ in the measurement region. By removing stave 16 and doubling stave 8 , the response improves dramatically so that the response varies between -1.5 and $0 \mathrm{~dB}$. When stave-beam renormalization is added, the response varies between -0.5 and $0.5 \mathrm{~dB}$ in the measurement region. Defining the beam width by the $-2 \mathrm{~dB}$ points, the width of the compensated beam pattern is about $85^{\circ}$. This is somewhat larger than the desired $77^{\circ}$, which is not surprising since the earlier analysis indicated a boost in power. This slight increase in size is not much of a problem from a measurement standpoint, but will cause a small decrease in array gain. The phases of the beam responses are shown in Fig. 11. As can be seen, the phase is non-zero for all cases because of the lack of array symmetry. For this example, there is about $40^{\circ}$ of error in the compensated response, but only at the edges of the measurement region. This can cause about $0.5 \mathrm{~dB}$ of signal loss where aperture 1 and 2 merge spectrally in the time series output [4]. However, this is considering a severe case because this effect has been shown to be greatest at the edges of the large horizontal beams such as those shown in Fig. 9. Also, the error is increased here because stave 16 and stave 8 are heavily weighted for the frequency and horizontal steering presented

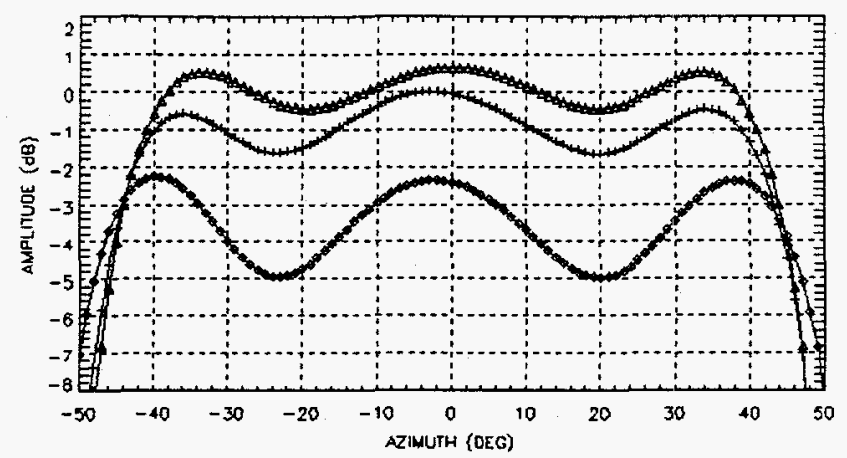

Fig. 9. Azimuthal beam responses for 34 missing sensors. $(\diamond)$ uncompensated, $(+)$ stave doubling: stave 16 removed and stave 8 doubled, $(\Delta)$ stave doubling and stave-beam renormalization.

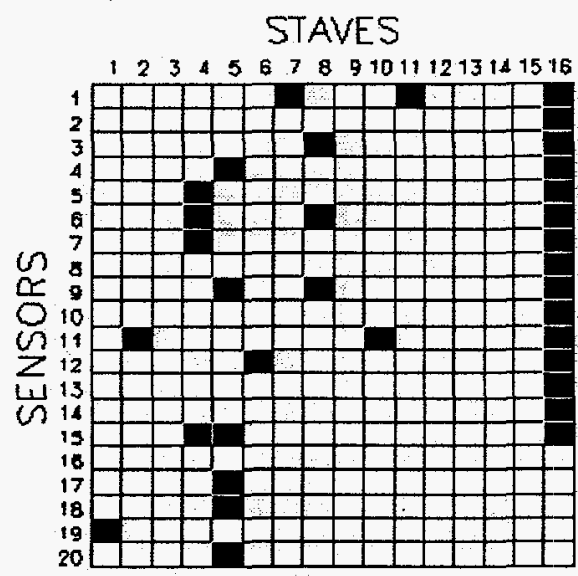

Fig. 10. Missing sensor grid for Fig. 9.

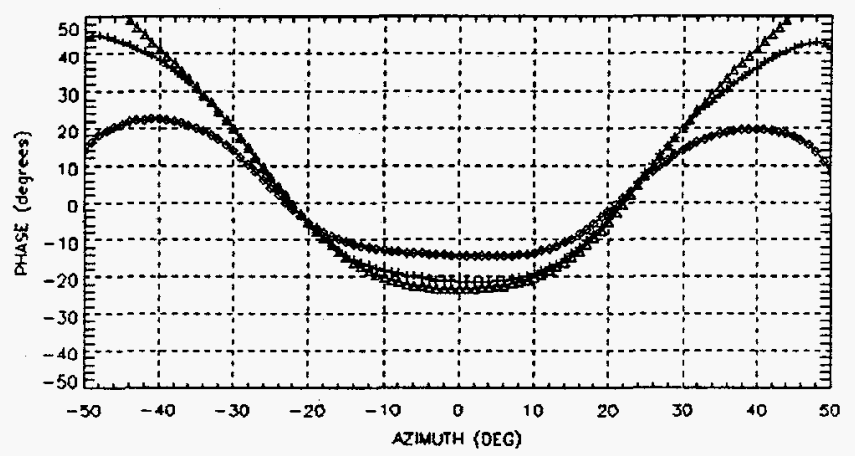

Fig. 11. Azimuthal phase responses corresponding to Fig. 9.

$(\diamond)$ uncompensated, $(+)$ stave doubling: stave 16 removed and stave 8 doubled, $(\Delta)$ stave doubling and stave-beam renormalization.

\section{ARRAY GAIN}

As mentioned before, the array gain can change when sensors are missing. As is the case for measurement accuracy, the change in array gain is dependent on the frequency, beam width, and steering. Table 1 shows some examples of how measurement error and array 
TABLE 1

Comparison of amplitude errors and array gains for different azimuthal steering angles and levels of compensation. The vertical and horizontal beam widths are $6^{\circ}$ and $18^{\circ}$. The vertical and horizontal sensor spacings are $0.68 \lambda$ and $0.63 \lambda$. The vertical steering is on the horizon, and the azimuthal $0^{\circ}$ reference is stave 16. The locations of the missing sensors are indicated by Fig. 10.

\begin{tabular}{ccccccc}
\hline & \multicolumn{2}{c}{ Azimuthal steering $=0^{\circ}$} & \multicolumn{2}{c}{ Azimuthal steering $=45^{\circ}$} & \multicolumn{2}{c}{ Azimuthal steering $=90^{\circ}$} \\
\hline Compensation & $\begin{array}{c}\text { Beam center } \\
\text { error }(\mathrm{dB})\end{array}$ & $\begin{array}{c}\text { Array gain } \\
(\mathrm{dB})\end{array}$ & $\begin{array}{c}\text { Beam center } \\
\text { error }(\mathrm{dB})\end{array}$ & $\begin{array}{c}\text { Array gain } \\
(\mathrm{dB})\end{array}$ & $\begin{array}{c}\text { Beam center } \\
\text { error }(\mathrm{dB})\end{array}$ & $\begin{array}{c}\text { Array gain } \\
(\mathrm{dB})\end{array}$ \\
\hline All good sensors & 0.0 & 33.7 & 0.0 & 33.7 & 0.0 & 33.7 \\
No compensation & -2.2 & 27.3 & -1.5 & 27.3 & -0.8 & 29.4 \\
Stave doubling & -0.8 & 24.8 & -0.8 & 26.7 & -0.7 & 29.3 \\
$\begin{array}{c}\text { Stave-beam } \\
\text { renormalization and } \\
\text { stave doubling }\end{array}$ & 0.0 & 24.1 & 0.0 & 26.3 & 0.0 & 28.6 \\
\hline
\end{tabular}

gain are affected by missing sensors and compensation. The table indicates performance for only one scenario, in which the sensor loss is rather severe. In the table, the "beam center error" is the offset of the beam pattern center from $0 \mathrm{~dB}$. The vertical and horizontal beam widths are $6^{\circ}$ and $18^{\circ}$. Since these beams have small width, they do not have ripple in the measurement region, and the error can reasonably be quantified by one number.

The table's examples show that missing sensors can decrease array gain and that the compensation can decrease the gain by a small amount. It also indicates these changes are a function of horizontal steering. As the azimuthal angle changes, the staves are weighted differently. This shows that some angles may have a higher signal-to-noise ratio than others; however, measurements above the noise threshold will remain accurate.

\section{CONCLUSIONS}

The approaches described here are a means of achieving accurate beamformed measurements using an array with some missing sensors. The approaches do not require modifying the existing coefficients. The methods include scaling individual staves and doubling certain staves so that the signals being measured have the appropriate levels. As has been indicated, array gain may change based on the array geometry, frequency, beam width and steering. However, the compensation makes the sound pressure level measurements reliable as these factors change.

The ideas presented were investigated using beam pattern simulations and analysis. After developing the techniques in simulations, stave-beam renormalization and stave doubling were implemented in the HGA beamformer system and used to compensate underwater acoustic measurements. The methods were tested using a controlled underwater source, and the technique was shown to correct the measurement inaccuracies that arose when multiple sensors were removed.

Because of the HGA beamformer system's unique design and needs, renormalizing and doubling conjugate sub-arrays are very beneficial. The system uses a large number of pre-calculated coefficients to acquire accurate acoustic measurement levels for moving angular regions of constant width. Also, the system uses array symmetry to handle the many hydrophones and possible steering angles. There may be other applications in which these compensation approaches are helpful. In particular, applications that might benefit are real-time systems that process data from multiple sub-arrays where sensor loss is possible and the response accuracy is critical.

\section{ACKNOWLEDGMENTS}

The authors would like to thank Rodney Cagle for creating the array analysis software which provides the core of the beam pattern simulation tool.

\section{REFERENCES}

[1] D. H. Johnson and D. E. Dudgeon, Array Signal Processing. Englewood Cliffs, NJ: Prentice-Hall, 1993.

[2] W. S. Burdic, Underwater Acoustic System Analysis. Englewood Cliffs, NJ: Prentice-Hall, 1991.

[3] A. V. Oppenheim and R. W. Schafer, Discrete-Time Signal Processing. Englewood Cliffs, NJ: Prentice-Hall, 1989.

[4] R. W. Tucker, Jr., "Reconstruction of a Composite Time Series Output for a Multiple-Aperture Frequency-Domain Beamformer," master's thesis, University of Tennessee, Knoxville, May 1995. 